Documentation et bibliothèques

DOCUMENTATION BIBLIOTHEQUES

\title{
DSI/LAVAL - Organisation et gestion d'un service automatisé de recherches bibliographiques dans une bibliothèque universitaire
}

\section{Gilles Deschatelets et Claude Bonnelly}

Volume 22, numéro 1, mars 1976

URI : https://id.erudit.org/iderudit/1055335ar

DOI : https://doi.org/10.7202/1055335ar

Aller au sommaire du numéro

Éditeur(s)

Association pour l'avancement des sciences et des techniques de la documentation (ASTED)

ISSN

0315-2340 (imprimé)

2291-8949 (numérique)

Découvrir la revue

Citer cet article

Deschatelets, G. \& Bonnelly, C. (1976). DSI/LAVAL — Organisation et gestion d'un service automatisé de recherches bibliographiques dans une bibliothèque universitaire. Documentation et bibliothèques, 22(1), 17-28.

https://doi.org/10.7202/1055335ar
Résumé de l'article

Consciente des nouveaux besoins documentaires de la recherche et de l'enseignement, sensiblisée aux ressources et aux techniques nouvelles de repérage et de transfert de l'information, la bibliothèque de l'Université Laval a créé le service DSI/LAVAL. Les auteurs racontent l'évolution de ce service et expliquent son « approvisionnement " de même que son fonctionnement.
Tous droits réservés (c) Association pour l'avancement des sciences et des techniques de la documentation (ASTED), 1976
Ce document est protégé par la loi sur le droit d'auteur. L'utilisation des services d'Érudit (y compris la reproduction) est assujettie à sa politique d'utilisation que vous pouvez consulter en ligne.

https://apropos.erudit.org/fr/usagers/politique-dutilisation/ 


\title{
DSI/LAVAL - Organisation et gestion d'un service automatisé de recherches bibliographiques dans une bibliothèque universitaire
}

\author{
Gilles Deschatelets \\ Claude Bonnelly \\ Coordonnateurs DSI/LAVAL \\ Université Laval \\ Québec
}

Consciente des nouveaux besoins documentaires de la recherche et de l'enseignement, sensiblisée aux ressources et aux techniques nouvelles de repérage et de transfert de l'information, la bibliothèque de l'Université Laval a créé le service DSI/LAVAL. Les auteurs racontent l'évolution de ce service et expliquent son "approvisionnement» de même que son fonctionnement.

Being conscious of modern documentation requirements in research and teaching, and aware of current sources and techniques for the tracing and transfer of information, the Library of Laval University has set up the DSI/LAVAL service. The evolution of this service is described by the authors, who also explain its "stocking up" as well as its operation.

Dándose cuenta de la necesidad documental de la busca y enseñanza, sensibilisada con los recursos y nuevas técnicas de busca e intercambio de información, la biblioteca universitaria de Laval ha creado el servicio DSI/LAVAL. Los autores hablan de la evolución de esta servicio y explican su "provisión" y funcionamiento.

On reconnaît généralement deux principaux rôles à la fonction de référence dans une bibliothèque. Ces rôles sont adéquatement décrits dans un récent projet de normes publié par l'American Library Association:

\footnotetext{
"The distinguishing feature of reference services is that it specifically ensures the optimum uses of information resources through substantive interaction with the users on direct and indirect levels cited below:

\begin{abstract}
A. Reference or information services consist of personal assistance provided to users in pursuit of information... The feature of information service, irrespective of its level or its intensity, is to provide an end-product in terms of information sought by the user.

B. Formal and informal instruction in the use of the library or information center and its resources..."
\end{abstract}

Selon la nature et le niveau des bibliothèques et selon les besoins spécifiques de leur clientèle, l'une de ces activités aura prédomi- nance sur l'autre. Mais il va sans dire que, dans les bibliothèques universitaires, spécialisées ou de recherche, le rôle de «fournisseur direct d'information» prend de plus en plus le pas sur le rôle d'initiateur aux ressources. Ce phénomène est facile à comprendre.

Des statistiques aujourd'hui célèbres ${ }^{2}$ montrent l'explosion spectaculaire qu'a connue, depuis quelques décennies, le monde de la documentation et ce dans tous les domaines. Le chercheur, même avec la meilleure volonté, n'a plus le temps de faire lui-même l'inventaire de toutes les ressources disponibles. Par ailleurs, ce même chercheur est pris dans l'engrenage de plus en plus étourdissant du monde de la recherche. Les projets de recherche, tout comme la documentation, naissent et se développent à un rythme accéléré; s'il veut être de la course, il doit en épouser le rythme.

Pour faire face de façon satisfaisante à ses problèmes documentaires, le chercheur doit donc consulter de plus en plus le bibliothécaire, et il exige de lui non plus qu'il soit un bon

2. Jean-Paul Trystram, La documentation automatique, Paris, Dunod, 1971, p. 6 


\begin{tabular}{|l|l|}
\hline ANNEE & $\begin{array}{c}\text { NOMBRE DE PÉRIODIQUES } \\
\text { SCIENTIFIQUES DANS LE MONDE }\end{array}$ \\
\hline 1750 & 10 \\
\hline 1800 & 100 \\
\hline 1850 & 1,000 \\
\hline 1900 & 10,000 \\
\hline 1970 & 100,000 (30 millions de pages imprimées) \\
\hline 2000 & $1,000,000$ \\
\hline
\end{tabular}

TABLEAU 1: Croissance des publications scientifiques

guide, mais qu'il lui fournisse, dans les meilleurs délais, un accès facile aux informations dont il a ou aura besoin.

C'est là une nouvelle exigence à laquelle les bibliothèques doivent constamment faire face. II est assez révélateur de constater que, dans les normes énoncées précédemment, le rôle de fournisseur d'information est mentionné en premier lieu, alors que dans les normes de 1961, il était en second lieu ${ }^{3}$.

Le bibliothécaire d'aujourd'hui doit donc utiliser tous les moyens disponibles pour répondre à ces besoins nouveaux relatifs au transfert de l'information: diffusion sélective de l'information, recherche bibliographique par ordinateur en mode différé et conversationnel, exploitation, gestion, création de banques de données, d'information ou documentaires de toutes sortes.

F. Wilfrid Lancaster parle de l' «information services librarian" et il le définit comme "a professional librarian who specializes in the exploitation of machine-readable files" ${ }^{4}$.

La bibliothèque de l'Université Laval, consciente d'une part, de ses responsabilités face à ces nouveaux besoins documentaires de la recherche et de l'enseignement et, d'autre part, sensibilisée à ces ressources et ces techniques nouvelles de repérage de l'information, a décidé d'y souscrire de façon rationnelle et planifiée. Elle a donc développé le service DSI/LAVAL.

3. "Reference standards», $R Q$, vol. 1, no. 2 (June 1961), 1-2.

4. "Twelfth annual clinic on library applications of data processing, Champaign, III, April 27-30, 1975», Program.

\section{Historique}

C'est en 1967 que la bibliothèque de l'Université Laval a commencé à exploiter les services externes de diffusion sélective de l'information. Elle offrit d'abord l'accès à la banque américaine A.S.C.A. (Automatic Subject Citation Alert) de I'Institute for Scientific Information de Philadelphie.

Avec la création du CAN/SDI en 1969, l'éventail des banques offertes s'accrut considérablement.

En mai 1971, GESYDAMS (Groupe d'Exploitation des Systèmes Documentaires Automatiques en Médecine et en Sciences) était formé. L'équipe, composée de bibliothécaires de référence et d'informatistes, avait le mandat de promouvoir l'utilisation et de faciliter l'exploitation des banques d'information. C'est surtout grâce à cette équipe que le service de D.S.I. a connu, à l'Université Laval, un essor si remarquable.

En juin 1973, cependant, l'équipe fut dissoute et la D.S.I. fut intégrée au service de référence de la bibliothèque, sous la coordination de deux bibliothécaires de ce secteur. Le service devint DSI/LAVAL.

Deux éléments nouveaux ont eu, depuis ce temps, un impact considérable sur l'organisation et le développement de DSI/LAVAL: la disponibilité de plusieurs nouvelles banques dans tous les domaines et l'essor fantastique du mode conversationnel. Tous ces facteurs nous ont donc amenés à mettre en place une structure plus raffinée, tant au niveau fonctionnel qu'au niveau administratif.

\section{Structure fonctionnelle}

Au plan de l'organisation fonctionnelle du service, la bibliothèque s'est définie, dès le début, comme "consommatrice" de services d'information, c'est-à-dire qu'elle a décidé de s'orienter davantage vers l'exploitation de services externes que vers la création ou la gestion de banques d'information.

Dans cette optique, le rôle du bibliothécaire de référence en est donc un d'intermédiaire local entre l'usager et toute une gamme de services et de systèmes documentaires qui lui seraient autrement difficilement accessibles. Nous avons alors apporté une distinction fondée sur le "contenu » des systèmes d'information accessibles.

C'est ainsi que nous avons distingué, 
d'après leur contenu, les «banques de données", les "banques d'informations bibliographiques» et les "banques documentaires».

\section{1) Banques de données:}

ces banques contiennent l'information directe, l'information reçue ne référant à aucune autre source et étant constituée d'éléments ou de données brutes n'ayant pas à être sortis d'un contexte. Les banques de données sont de deux types: "factuelles» (ex.: SSIE, UNION, banque de produits québécois du CRIQ, etc.) et "numériques» (ex.: CANSIM).

\section{2) Banques d'informations bibliographiques:}

ces banques sont constituées de références bibliographiques; elles contiennent donc de l'information indirecte, l'information reçue référant à une autre source (le document) qui doit être analysée pour en tirer les éléments d'information désirés. En général, ces banques correspondent aux divers index imprimés: Biological Abstracts, Index Medicus, Psychological Abstracts, etc.

\section{3) Banques documentaires:}

ces banques sont constituées des documents eux-mêmes; elles contiennent donc de l'information directe et indirecte; directe, parce que l'usager reçoit non pas une référence mais la source elle-même, c'est-à-dire le texte complet du document; indirecte, parce qu'il doit encore l'analyser pour en tirer les éléments d'information voulus. Ces banques correspondent, la plupart du temps, à des fonds documentaires spécialisés, comme MODUL/DEPLOI.

La seconde distinction apportée concerne le mode d'accès à ces diverses catégories de banques d'information: mode différé (accès indirect) et mode conversationnel (accès direct).

\section{1) Mode différé}

Le mode différé suppose la rédaction d'un profil d'intérêt codifié, selon des normes différentes pour chaque système, et transmis par courrier à l'organisme responsable de son exploitation. Pour faciliter la tâche des bibliothécaires-rédacteurs, il a fallu établir un formulaire unique de rédaction de profil; le contenu de ce formulaire est traduit par les coordonnateurs selon les normes spécifiques du système interrogé, s'il y a lieu.

DSI/LAVAL a actuellement des contacts avec 14 centres ou organismes fournisseurs de services documentaires (canadiens, américains, européens) et offre ainsi l'accès à 33 banques d'informations différentes en mode différé (voir Tableau 2).

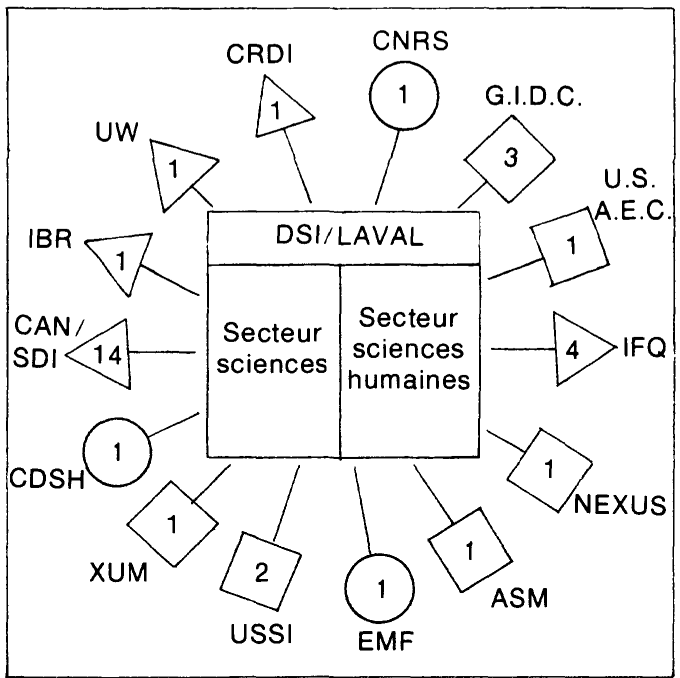

TABLEAU 2: DSI/LAVAL: Mode différé

N.B.: Le chiffre inscrit dans chaque figure indique le nombre de banques de chaque centre exploitées en mode différé par DSI/LAVAL.

Légende:

$$
\begin{aligned}
& \triangle \text { - centre canaaien } \\
& \triangle \text { - centre européen } \\
& \square \text { - centre américain }
\end{aligned}
$$

\section{2) Mode conversationnel}

Le mode conversationnel suppose l'interrogation immédiate d'une banque à partir d'un terminal. DSI/LAVAL possède maintenant quatre terminaux, deux à la bibliothèque scientifique, et deux à la bibliothèque générale. Plusieurs systèmes étant multidisciplinaires, nous avons organisé les modes de communication de façon à ce que tous les systèmes soient accessibles à l'une et l'autre des bibliothèques. En outre, pour simplifier le contrôle administratif et accrôitre l'efficacité des recherches, il a été établi, comme politique, qu'un bibliothé- 
caire devait être responsable de chaque recherche au terminal, de préférence en présence de l'usager. De plus, pour lui faciliter la tâche, nous rédigeons, s'il y a lieu, pour chaque système un guide d'utilisation en français.

Jusqu'à maintenant, DSI/LAVAL a signé des ententes avec dix organismes pourvoyeurs de services d'information en mode conversationnel. Nous exploitons, en outre, un certain nombre de fichiers internes à la bibliothèque. L'ensemble de ces services permet d'offrir l'accès à quelque 52 fichiers externes différents (voir Tableau 3), en plus des divers fichiers de la bibliothèque.

Ainsi, une utilisation rationnelle de ces deux modes d'accès permet d'offrir, aux usagers, un ensemble de 66 banques ou fichiers différents, couvrant pratiquement tous les domaines de la connaissance (voir liste, Tableau 4, Annexe I).
Cela permet, en outre, d'offrir une grande variété de services allant de la recherche rétrospective à la mise au courant. La politique, en ce domaine, est de localiser, pour chacune des banques accessibles, un centre ou un organisme offrant un service de recherches rétrospectives et de mise au courant. La tendance actuelle, dans les services de même nature que DSI/LAVAL, semble être l'utilisation du mode différé pour la mise au courant et du mode conversationnel pour les recherches rétrospectives, ce qui semble tout à fait logique du point de vue de l'efficacité, compte tenu des avantages et des inconvénients de chacun de ces modes d'accès.

\section{Structure administrative}

Nous avons mis au point une structure administrative relativement simple et efficace, compte tenu de la structure fonctionnelle; le principal objectif est de favoriser l'utilisation

TABLEAU 3: DSI/LAVAL: Mode conversationnel

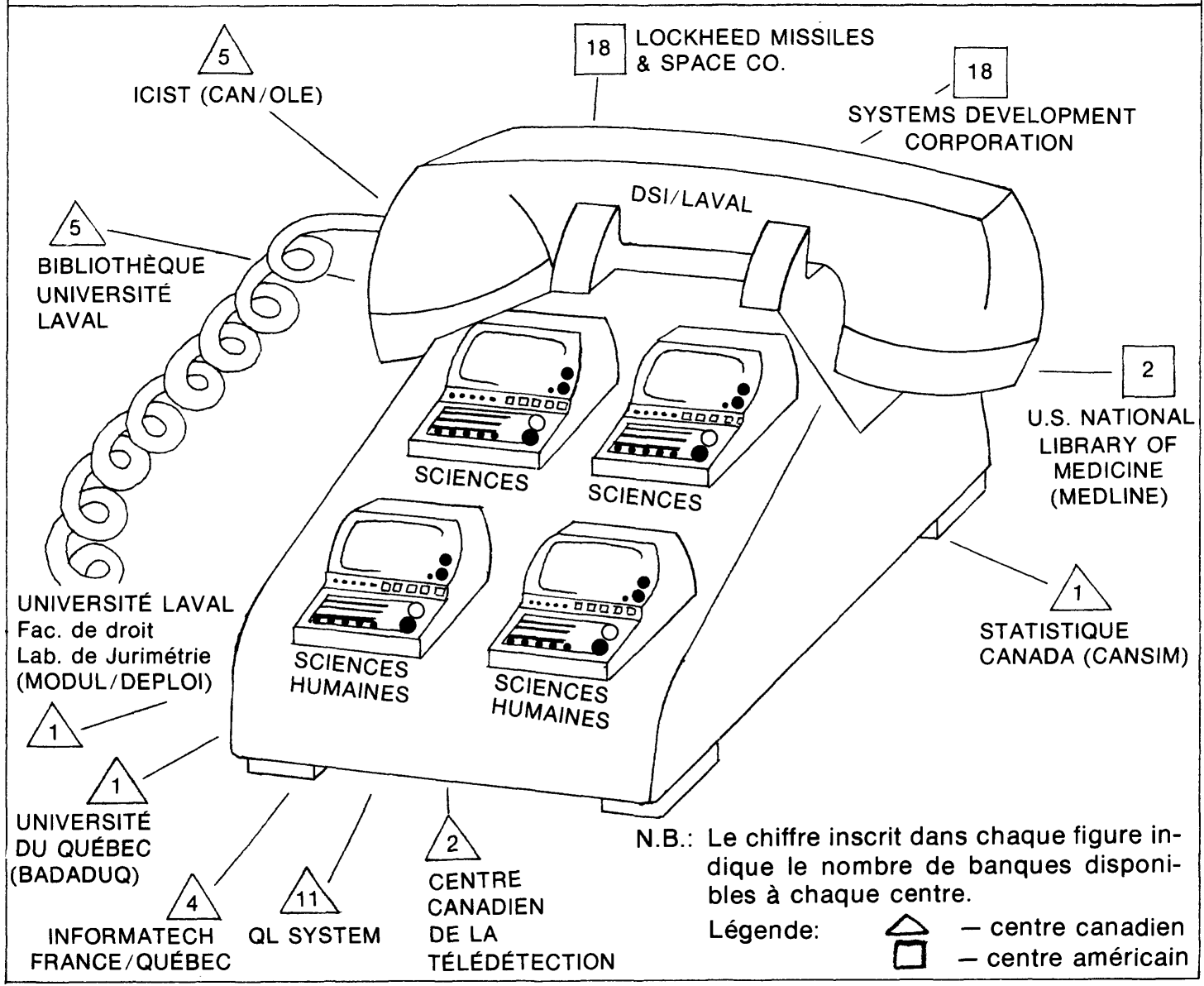


des services, tout en évitant, autant que possible, à l'usager, les désagréments de l'aspect administratif du service qui auraient pu, facilement, devenir très lourds.

Nous avons donc adopté, au départ, le principe suivant: c'est la bibliothèque qui défraie, à même le budget de DSI/LAVAL, le coût de toutes les recherches effectuées par les usagers, que ce soit en mode différé ou en mode conversationnel. Elle se fait rembourser, par la suite, s'il y a lieu, selon l'application de la politique de tarification mise au point. Cette politique est la suivante:

en mode différé: en principe, le coût de la recherche est assumé entièrement par l'usager; cependant DSI/LAVAL défraie $\$ 40.00$ pour chaque banque exploitée, pendant la première année de fonctionnement de tout nouveau profil de mise au courant; cela ne s'applique qu'à la communauté universitaire.

en mode conversationnel: le coût d'une recherche a été fixé à $\$ 3.00$ par banque ou fichier consulté pour les membres de la communauté universitaire et à $\$ 10.00$ pour les usagers de l'extérieur.

Tout l'aspect administratif du service relève des coordonnateurs et de l'adjoint administratif de la bibliothèque. II existe des formules de contrat que les bibliothécaires n'ont qu'à faire signer par les usagers et à remettre à l'un des coordonnateurs.

La politique de tarification prévoit également que le coût des premières recherches effectuées dans une nouvelle banque sera assumé entièrement par DSI/LAVAL.

Enfin, chacun des bibliothécaires de l'équipe DSI/LAVAL possède son propre profil d'intérêt, ce qui lui permet, d'une part, de se familiariser avec les techniques de gestion de profil et, d'autre part, de connaître plus en profondeur le contenu de la ou des banques interrogées. Tous les coûts de ces profils sont, bien sûr, assumés par DSI/LAVAL.

\section{Formation des bibliothécaires}

L'équipe DSI/LAVAL se compose actuellement de 26 bibliothécaires de services de référence et de développement des collections. Chaque bibliothécaire est spécialisé dans une discipline particulière, s'occupant ainsi des relations avec le ou les départements ou la ou les facultés relevant de sa discipline; il connaît les diverses techniques bibliothéconomiques relatives au repérage «traditionnel» de la documentation; en outre, sa participation à l'équipe DSI/LAVAL suppose une formation spéciale en science de l'information: il doit maîtriser les techniques de repérage automatisé de l'information.

Ce dernier aspect de la formation des bibliothécaires était précisément la responsabilité des coordonnateurs. La réalisation de cet objectif a nécessité le programme suivant:

$1^{\circ}$ rédaction d'un manuel de références exhaustif sur le service DSI/LAVAL

$2^{\circ}$ une semaine de stages pratiques sur la rédaction et la gestion de profils d'intérêts, complétée de plusieurs séances d'entraînement pour les recherches au terminal;

$3^{\circ}$ la rédaction et la gestion, par chaque bibliothécaire, de «son» profil d'intérêt, occasionnant plusieurs rencontres personnelles entre chaque bibliothécaire et les coordonnateurs.

L'application de ce programme a occasionné, chez quelques bibliothécaires, une certaine "réticence», voire même une certaine "crainte» face à ces techniques nouvelles; cette appréhension semble cependant vaincue et si tous les bibliothécaires ne s'impliquent pas nécessairement de façon active, dans ce nouveau service du moins, nous croyons néanmoins qu'ils n'hésiteront pas à le faire tôt ou tard, pour répondre aux besoins de leur clientèle et pour s'intégrer davantage à la recherche et à l'enseignement de leurs milieux respectifs.

\section{Services offerts}

Le concept d'une équipe de bibliothécaires de référence, spécialisés dans un domaine particulier et maîtrisant à la fois diverses techniques de la bibliothéconomie et des sciences de l'information, permet d'offrir aux usagers une grande variété de services pour le repérage et la diffusion de l'information:

- service-conseil: choix des média (sources, techniques, approches) à utiliser pour répondre aux besoins précis de chaque usager;

- service de rédaction, gestion, annulation des profils d'intérêts;

- service de recherches par terminal;

- service d'évaluation (analyse statistique) des recherches ou des profils d'intérêts: 
- absorption, par la bibliothèque, d'une partie importante du coût d'exploitation de ses divers services.

Cette structure particulière du service DSI/LAVAL présente donc plusieurs avantages non seulement pour la bibliothèque (extension de la référence, rôle plus actif dans la recherche et l'enseignement, meilleure connaissance des besoins documentaires du milieu, exploitation accrue de ses propres ressources, etc.), mais également pour les organismes fournisseurs (communications facilitées, meilleur "feedback», meilleure connaissance des besoins) et, bien sûr, pour l'usager (économie de temps, documentation plus complète, service personnalisé adapté à ses besoins, recherche plus souple, information récente, etc.).

Nous avons établi un programme de publicité qui comprend: une brochure expliquant l'ensemble du service, un feuillet publicitaire pour chacune des banques offertes, une liste des prix en vigueur, une brochure traitant des services en mode conversationnel, une série d'articles dans le journal de l'Université ( $A u$ fil des événements), une lettre à tous les chercheurs, professeurs et étudiants de maîtrise et de doctorat de l'Université et un diaporama.

En outre, chaque bibliothécaire doit veiller à promouvoir le service dans son secteur par des contacts personnels et une présence «active" à des réunions, conférences et rencontres.

\section{Conclusion}

Bien que relativement récente, cette expérience de gestion et d'exploitation d'un service comme DSI/LAVAL a démontré la nécessité d'une bonne planification initiale, non seulement au niveau du service comme tel, mais également dans l'optique d'une politique documentaire globale de la bibliothèque. La bibliothèque représente, à toutes fins pratiques, un système documentaire intégré dans lequel tous les modules sont étroitement reliés. DSI/LAVAL n'échappe pas à cette interconnexion des services; au contraire, sa situation même, dans la chaîne documentaire de la bibliothèque, de lien direct et immédiat avec l'usager en fait peut-être l'un des services ayant le plus d'affinités et d'influence sur chacun des autres modules: développement des collections, acquisitions, traitement, prêt entre bibliothèques, circulation, etc.

La référence doit être redéfinie dans les bibliothèques universitaires. Traditionnellement, elle était considérée comme l'étape ter- minale de la chaîne documentaire selon le schéma linéaire: acquisition, traitement, exploitation (voir Tableau 4). Le processus est modifié; il peut être désormais défini comme un cycle documentaire (voir Tableau 5) dans lequel on retrouve une interpénétration de tous les modules. Le schéma défini par Abraham Moles pour décrire le processus des communications s'applique parfaitement au monde de la documentation.

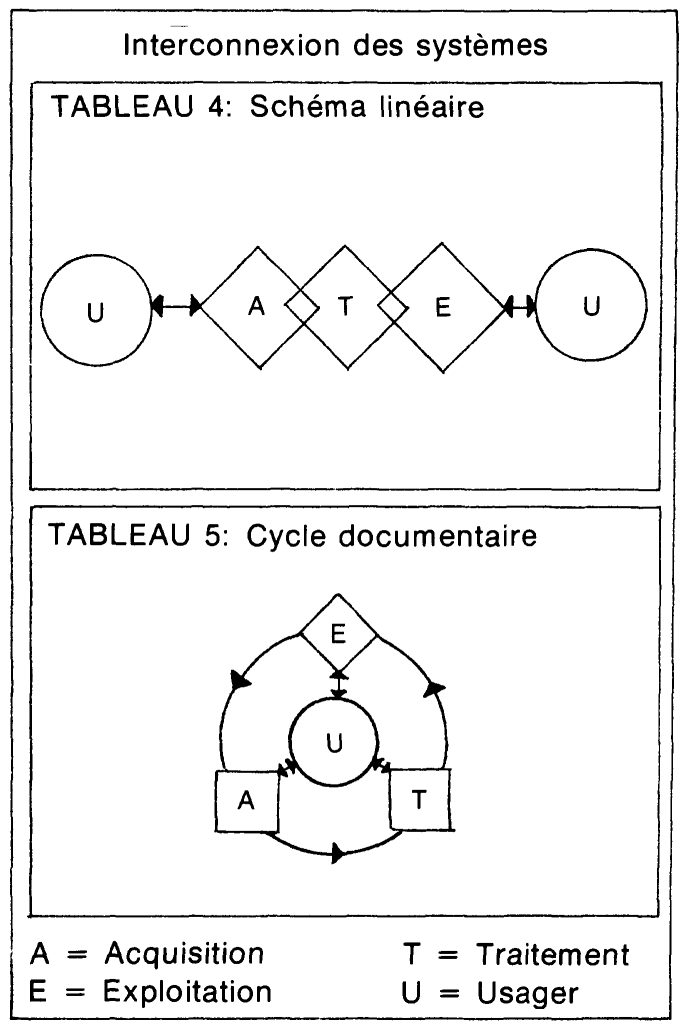

Cette redéfinition du module "exploitation» a donc une portée considérable sur l'orientation future des bibliothèques universitaires. Grâce à l'ordinateur et à l'essor prodigieux des télécommunications, le module «exploitation" n'est plus limité aux seules ressources de la bibliothèque. Le concept de «téléréférence" a fait son apparition. Des besoins nouveaux sont ainsi créés et le "feedback» ne tarde pas à se manifester: I'usager veut obtenir rapidement les documents signalés; il peut avoir besoin de services de traduction, si ces documents ne sont pas dans une langue qu'il maîtrise; il peut également requérir de l'aide pour contrôler, organiser et exploiter la masse d'information qu'il reçoit.

La bibliothèque doit donc voir à satisfaire tous ces nouveaux besoins qu'elle a ainsi

5. Abraham Moles, Sociodynamique de la culture, Paris, Mouton, 1967, p. 95 
engendrés; développement des collections (à partir du module exploitation), prêt entre bibliothèques, traitement (primaire, secondaire, tertiaire), exploitation, création de réseaux documentaires, voilà autant de moyens à sa disposition pour prévoir et satisfaire ces nouveaux besoins de l'usager, à la condition que ces éléments soient intégrés dans une politique globale.
L'influence de l'usager sur les politiques et l'orientation de la bibliothèque est considérablement accrue. Et nous croyons que cette tendance confirme merveilleusement le but essentiel de toute bibliothèque: le service à l'usager.

\begin{tabular}{|c|c|c|c|c|}
\hline \multicolumn{5}{|c|}{ ANNEXE I } \\
\hline \multirow{2}{*}{ BANQUE } & \multicolumn{2}{|c|}{ SERVICE OFFERT } & \multirow{2}{*}{$\begin{array}{c}\text { MODE } \\
\text { D'ACCÉS }\end{array}$} & \multirow{2}{*}{$\begin{array}{l}\text { ORGANISME } \\
\text { FOURNISSEUR }\end{array}$} \\
\hline & Mac & Retro & & \\
\hline \multirow{3}{*}{$A B I / I N F O R M$} & & $1971+$ & C & LOCKHEED \\
\hline & & $1971+$ & C & SDC \\
\hline & & $1971+$ & C & QLS \\
\hline AIM / ARM & & $1966+$ & C & LOCKHEED \\
\hline \multirow{2}{*}{ AGRIDOQ } & $12 / a n$ & $1974+$ & $\mathrm{D}$ & $\mathrm{IF}-\mathrm{Q}$ \\
\hline & & 1972 & C & $\mathrm{IF}-\mathrm{Q}$ \\
\hline APILIT & & $1964+$ & C & SDC \\
\hline APIPAT & & $1964+$ & C & SDC \\
\hline ASI & & $1974+$ & C & SDC \\
\hline BADADUQ & & & C & U du Q \\
\hline \multirow{2}{*}{ BAP } & $36 / a n$ & & $\mathrm{D}$ & CAN/SDI \\
\hline & & $1972+$ & C & CAN/OLE \\
\hline BASIS & & $1834+$ & $\mathrm{D}$ & NEXUS CORP. \\
\hline \multirow{4}{*}{ CAC } & $52 /$ an & & $D$ & CAN/SDI \\
\hline & & $1972+$ & C & LOCKHEED \\
\hline & & $1970+$ & C & SDC \\
\hline & & $1973+$ & C & CAN/OLE \\
\hline CCALINE & & $1967+$ & C & N.L.M. \\
\hline \multirow{3}{*}{ CAIN/NAL } & $12 / a n$ & & D & CAN/SDI \\
\hline & & $1970+$ & C & LOCKHEED \\
\hline & & $1970+$ & C & SDC \\
\hline CANSIM & & $1946+$ & C & S.M.A. \\
\hline C.I.S. & & $1970+$ & C & SDC \\
\hline CLAIMS & & $1950+$ & C & LOCKHEED \\
\hline
\end{tabular}




\begin{tabular}{|c|c|c|c|c|}
\hline \multirow{2}{*}{ BANQUE } & \multicolumn{2}{|c|}{ SERVICE OFFERT } & \multirow{2}{*}{$\begin{array}{c}\text { MODE } \\
\text { D'ACCEES }\end{array}$} & \multirow{2}{*}{$\begin{array}{l}\text { ORGANISME } \\
\text { FOURNISSEUR }\end{array}$} \\
\hline & Mac & Retro & & \\
\hline \multirow{4}{*}{ COMPENDEX } & $12 / a n$ & & $\mathrm{D}$ & CAN/SDI \\
\hline & & $1969+$ & $\mathrm{D}$ & CAN/OLE \\
\hline & & $1973+$ & $c$ & LOCKHEED \\
\hline & & $1970+$ & C & SDC \\
\hline \multirow{2}{*}{ CT } & $26 /$ an & & $\mathrm{D}$ & CAN/SDI \\
\hline & & $1962-68$ & $\mathrm{D}$ & G.I.D.C. \\
\hline DATRIX & & $1861+$ & $\mathrm{D}$ & X.U.M. \\
\hline ECA & & $1966+$ & C & LOCKHEED \\
\hline ENV/WATDOC & & & C & QLS \\
\hline \multirow{3}{*}{ ERIC } & $12 /$ an & & $D$ & $\mathrm{CAN} / \mathrm{SDI}$ \\
\hline & & $1966+$ & C & S.D.C. \\
\hline & & $1966+$ & $\mathrm{C}$ & LOCKHEED \\
\hline EXCERPTA MEDICA & $52 /$ an & $1969+$ & $D$ & EMF \\
\hline GEOL & & $1965+$ & $\mathrm{D}$ & G.I.D.C. \\
\hline \multirow{2}{*}{ GEOREF } & $12 /$ an & & $\mathrm{D}$ & Can/SDI \\
\hline & & $1967+$ & c & SDC \\
\hline \multirow{3}{*}{ GRAN/NTIS } & $24 /$ an & & $D$ & $\mathrm{CAN} / \mathrm{SDI}$ \\
\hline & & $1970+$ & C & SDC \\
\hline & & $1964+$ & C & LOCKHEED \\
\hline HEEP & & & $\mathrm{C}$ & QLS \\
\hline IISS & & $1972+$ & C & $\mathrm{CCT}$ \\
\hline \multirow[t]{5}{*}{ INSPEC } & $24 /$ an & & D & CAN/SDI \\
\hline & & $1970+$ & C & LOCKHEED \\
\hline & & $1970+$ & $\mathrm{C}$ & LOCKHEED \\
\hline & & $1970+$ & C & LOCKHEED \\
\hline & & $1969+$ & C & CAN/OLE \\
\hline ISIS & & $1965+$ & D & CRDI \\
\hline \multirow{2}{*}{ MARC/LIBCON } & $52 / a n$ & & $\mathrm{D}$ & CAN/SDI \\
\hline & & $1965+$ & $\mathrm{C}$ & SDC \\
\hline MATRIX & & $1973+$ & $C$ & SDC \\
\hline \multirow[t]{2}{*}{ MEDLINE } & $12 /$ an & & $\mathrm{D}$ & CAN/SDI \\
\hline & & $1969+$ & C & N.L.M. \\
\hline
\end{tabular}




\begin{tabular}{|c|c|c|c|c|}
\hline \multirow{2}{*}{ BANQUE } & \multicolumn{2}{|c|}{ SERVICE OFFERT } & \multirow{2}{*}{$\begin{array}{c}\text { MODE } \\
\text { D'ACCES }\end{array}$} & \multirow{2}{*}{$\begin{array}{l}\text { ORGANISME } \\
\text { FOURNISSEUR }\end{array}$} \\
\hline & Mac & Retro & & \\
\hline \multirow{2}{*}{ METADEX } & $12 / a n$ & $1966+$ & $\mathrm{D}$ & ASM \\
\hline & & & C & QLS \\
\hline MODUL/DEPLOI & & $1964+$ & C & L.J.U.L. \\
\hline NEWS & & & C & QLS \\
\hline \multirow{2}{*}{ N.S.A. } & $24 / a n$ & $1966+$ & $\mathrm{D}$ & G.I.D.C. \\
\hline & & $1962+$ & $\mathrm{D}$ & U.S.A.E.C. \\
\hline$O A B$ & & $1970+$ & C & QLS \\
\hline \multirow{2}{*}{ PASAR } & $12 /$ an & & D & CAN/SDI \\
\hline & & $1967+$ & $\mathrm{C}$ & LOCKHEED \\
\hline PASCAL/SCI. \& TECH. & $10 /$ an & $1971+$ & $D$ & CNRS \\
\hline PASCAL/SCI. HUM. & $8 /$ an & $1973+$ & $\mathrm{D}$ & C.D.S.H. \\
\hline PATS (CMA/EMA) & & $1972+$ & C & LOCKHEED \\
\hline PATS (DOMESTIC STATISTICS) & & & C & LOCKHEED \\
\hline PATS (EIS PLANT FILE) & & & $\mathrm{C}$ & LOCKHEED \\
\hline PATS (F \& S INDEX) & & & C & LOCKHEED \\
\hline PATS (MKT ABST WKLY) & & & c & LOCKHEED \\
\hline PATS (SOURCE DIRECTORY) & & & c & LOCKHEED \\
\hline$P / E$ NEWS & & $1975+$ & C & SDC \\
\hline \multirow{2}{*}{ PLURIDOQ } & $10 /$ an & & $\mathrm{D}$ & $\mathrm{IF}-\mathrm{Q}$ \\
\hline & & $1973+$ & C & IF-Q \\
\hline POLLUTION/PAB & & $1970+$ & $\begin{array}{l}\mathrm{C} \\
\mathrm{C}\end{array}$ & $\begin{array}{l}\text { SDC } \\
\text { QLS }\end{array}$ \\
\hline RESORS & & $1951+$ & C & CCT \\
\hline RSC & & $1974+$ & c & QLS \\
\hline S.A. & & $1963+$ & $\mathrm{D}$ & GIDC \\
\hline SBC & & $1973+$ & $\mathrm{C}$ & QLS \\
\hline \multirow{2}{*}{$\mathrm{SCl}$ (SCI SEARCH) (section life science) } & $52 /$ an & & D & $\mathrm{CAN} / \mathrm{SDI}$ \\
\hline & & & C & S.D.C. \\
\hline SEARCH & & $1972+$ & C & SDC \\
\hline SIRLS & $3 / a n$ & $1963+$ & $D$ & U.W. \\
\hline SNB & & & C & QLS \\
\hline SPIN & $12 /$ an & $1970+$ & D & G.I.D.C. \\
\hline \multirow{2}{*}{$\mathrm{ssCl}$} & $2 / a n$ & & D & $\mathrm{CAN} / \mathrm{SDI}$ \\
\hline & & $1972+$ & $C$ & LOCKHEED \\
\hline
\end{tabular}




\begin{tabular}{|c|c|c|c|c|}
\hline \multirow{2}{*}{ BANQUE } & \multicolumn{2}{|c|}{ SERVICE OFFERT } & \multirow{2}{*}{$\begin{array}{l}\text { MODE } \\
\text { D'ACCEES }\end{array}$} & \multirow{2}{*}{$\begin{array}{l}\text { ORGANISME } \\
\text { FOURNISSEUR }\end{array}$} \\
\hline & Mac & Retro & & \\
\hline \multirow{3}{*}{ SSIE } & $12 / a n$ & & $\mathrm{D}$ & U.S.S.I. \\
\hline & & $1964+$ & $D$ & U.S.S.I. \\
\hline & & $1974+$ & C & S.D.C. \\
\hline SSIE/GRA & & $1964+$ & $D$ & U.S.S.I. \\
\hline SSIS & & $1968+$ & $D$ & I.B.R. \\
\hline \multirow{2}{*}{ TEXTILDOQ } & $12 / a n$ & & $D$ & $\mathrm{IF}-\mathrm{Q}$ \\
\hline & & $1969+$ & C & $\mathrm{IF}-\mathrm{Q}$ \\
\hline UNION & $\begin{array}{l}\text { Liste } \\
\text { à date }\end{array}$ & $\begin{array}{l}\text { Liste } \\
\text { à date }\end{array}$ & C & CAN/OLE \\
\hline \multirow{2}{*}{ URBADOQ } & $10 /$ an & & $D$ & $\mathrm{IF}-\mathrm{Q}$ \\
\hline & & $1973+$ & C & IF-Q \\
\hline WAA & & & C & QLS \\
\hline \multicolumn{5}{|l|}{$\begin{array}{l}\text { FICHIERS DE LA BIBLIOTHËQUE } \\
\text { DE L'UN. LAVAL: }\end{array}$} \\
\hline CIRCUL & \multirow{5}{*}{\multicolumn{2}{|c|}{$\begin{array}{l}\text { consultation } \\
\text { consultation } \\
\text { consultation } \\
\text { consultation } \\
\text { consultation }\end{array}$}} & $\mathrm{C}$ & C.T.I. \\
\hline TELECAT-COOP & & & C & UNIV. TORONTO \\
\hline CAPSUL & & & C & C.T.I. \\
\hline VEDUL & & & c & C.T.I. \\
\hline ACUL & & & C & C.T.I. \\
\hline
\end{tabular}

N.B.: Voir la liste des sigles en annexe II.

Légende: $\mathrm{C}=$ Conversationnel $\mathrm{D}=$ Différé

\section{ANNEXE II}

\section{EXPLICATION DES SIGLES UTILISÉS}

ABI/INFORM: Abstracted Business Information AIM/ARM: Abstracts of Instructional Materials/Abstracts of Research Materials

APILIT: American Petroleum Institute/Literature APIPAT: American Petroleum Institute/Patents ASI: American Statistical Index ASM: American Society for Metals BADADUQ: Banque de Données BAP: Biological Abstracts Previews

BASIS: Bibliographic Author or Subject Interactive Search

CAC: Chemical Abstracts Condensates

CCA: Computers and Control Abstracts

CCALINE: Cancer Chemotherapy on-line

CAIN/NAL: Cataloguing and Indexing/National Agricultural Library

CAN/OLE: Canadian On-Line Enquiry

CAN/SDI: Canadian Selective Dissemination of Information
CCT: Centre Canadien de la Télédétection

CDSH: Centre de Documentation Sciences Humaines

CIS: Congressional Information

CLAIMS: Class Code, Assignee, Index Method Search

CNRS: Centre National de la Recherche Scientifique

COMPENDEX: Computerized Engineering Index

CRDI: Centre de Recherche sur le Développement International

CT: Chemical Titles

CTI: Centre de Traitement de I'Information

DATRIX: Dissertation Abstracts

ECA: Exceptional Children: Abstracts

EEA: Electricity and Electronics Abstracts

EMF: Excerpta Medica Foundation

ENV/WATDON: Environment/Water Documentation

ERIC: Educational Resources Information Center 
GEOL: Geology (Bibliography of North American Geology)

GEOREF: Geological Reference File

GIDC: Georgia Information Dissemination Center

GRA/NTIS: Government Reports Announcements/National Technical Information Service

HEEP: Health Effects of Environmental Pollutants

IF-Q: Informatech France-Québec

IISS: Image Inventory Search and Summary

INSPEC: Information Service in Physics, Electrotechnology and Computers and Control

ISIS: Integrated Set of Information Systems

LJUL: Laboratoire de Jurimétrie de l'Université Laval

MARC/LIBCON: Machine-Readable Cataloguing/ Library of Congress

MEDLINE: Medical Literature Analysis and Retrieval System On-Line

METADEX: Metals Abstracts Index

MODUL/DEPLOI: Medium Ordinateur et Droit à I'Université Laval/Dépistage des Lois

NLM: National Library of Medicine

NSA: Nuclear Science Abstracts

OAB: Oceanic Abstracts

PA: Physics Abstracts

PAB: Pollution Abstracts

PASAR: Psychological Abstracts Search and Retrieval

PASCAL: Programme Appliqué à la Sélection et à la Compilation Automatique de la Littérature

PATS (CMA/EMA): Patents/Chemical and Electronics Market Abstracts

PATS (F \& S INDEX): Patents/Funk and Scott Index

PATS (MKT ABST WKLY): Patents/Market Abstracts Weekly

$P / E$ NEWS: Petroleum Energy News

QLS: QUIC Law System

RESORS: Remote Sensing On line Retrieval System

RSC: Revised Statutes of Canada

SA: Sociological Abstracts

SBC: Statutes of British Columbia

$\mathrm{SCl}$ : Science Citation Index

SDC: System Development Corporation

SEARCH: Systemized Excerpts Abstracts and Reviews of Chemical Headlines

SIRLS: System of Information Retrieval for Leisure and Sports

SMA: Société de Mathématiques Appliquées

SNB: Statutes of New Brunswick

SPIN: Searchable Physics Information Notices

SSCI: Social Science Citation Index

SSIE: Smithsonian Science Information Exchange

SSIS: Social Science Information System

USSI: United States Smithsonian Institution

UW: University of Waterloo

WAA: World Aluminium Abstracts

XUM: Xerox University Microfilm

\section{Bibliographie}

Bonnelly, C. «Banques de données et diffusion sélective de l'information en sciences humaines". in Stage pratique en informatique documentaire. Rapport. Montréal, 23-25 octobre 1974. Montréal, Corporation des bibliothécaires professionnels du Québec. 1975, 90-132

Bonnelly, C. "La référence automatisée dans les bibliothèques d'université et de recherche". Documentation et bibliothèques, vol. 19, no 1 (mars 1973), 26-33.

Bonnelly, C. et Deschatelets, G. "La Bibliothèque vous offre CANSIM: un système ultra-rapide de repérage de données statistiques". Au fil des événements, vol. 10, no 23 (février 1975), 7

Bonnelly, C. et Deschatelets, G. "La Bibliothèque vous offre MODUL /DEPLOI: un système rapide de repérage de l'information dans le domaine du droit statutaire québécois». Au fil des événements, vol. 10, no 29 (3 avril 1975), 15

Bonnelly, C. et Deschatelets, G. Guide d'utilisation du système CANSIM. Québec, Bibliothèque, Université Laval, 1975. 39 p.

Caron, G. Les banques en sciences sociales. Québec, Bibliothèque, Université Laval, 1973. 6 p. (miméographié)

Deschatelets, G. "L'ordinateur au service de l'information scientifique et médicale à l'Université Laval», La vie médicale au Canada français, vol. 1, no 2 (février 1974), 171-174.

Deschatelets, G. "Problèmes documentaires? Besoin d'information?" Hôpital d'aujourd'hui, vol. 18, no 5 (mai 1972), 35-37.

Deschatelets, G. et Bonnelly, C. "À la Bibliothèque: CAN/OLE - Un nouveau service ultra-rapide pour repérer de l'information". Au fil des événements, vol. 9, no 29 (mai 1974), 2

Deschatelets, G. et Bonnelly, C. CAN/OLE: évaluation de l'expérience pilote et recommandations à l'ICIST. Québec, Bibliothèque, Université Laval. Novembre 1974. 52 p. (Texte présenté au CAN/OLE).

Deschatelets, G. et Bonnelly, C. DSI/LAVAL. Québec, Bibliothèque, Université Laval, 1974. 12 p. (brochure publicitaire).

Deschatelets, G. et Bonnelly, C. Guide d'utilisation du système CAN/OLE. Québec, Bibliothèque, Université Laval, 1975. 24 p.

Deschatelets, G. et Bonnelly, C. Guide d'utilisation du système MEDLINE. Québec, Bibliothèque, Université Laval. (À paraître).

Deschatelets, G. et Bonnelly, C. Manuel des principes et des techniques de diffusion sélective de l'information à l'usage des bibliothécaires de l'Université Laval. Québec, Bibliothèque, Université Laval, 1975. 1309 p. 2 vol. (pagination variée). 


\section{VIENNENT DE PARAITTRE}

mentaire en mode conversationnel: CAN/OLE, MEDLINE", in Stage pratique en informatique documentaire. Rapport. Montréal, 23-25 octobre 1974. Montréal, Corporation des bibliothécaires professionnels du Québec, 1975, 166-204.

De Varennes, R. "Bibliothèques et bibliothécaires d'aujourd'hui et de demain à l'ère électronique", Bulletin de l'ACBLF, vol. 15, no 2 (juin 1969), 59-66.

Deschatelets, G. et Francœur, R. "GESYDAMS l'expérience de l'Université Laval dans la dissémination sélective de l'information», Documentation et bibliothèques, vol. 19, no 2 (juin 1973), 85-88

De Varennes, R. "Canadian library systems and networks: their planning and development in La Belle. Province (Québec)», Network, 15-18 sq, 197?

De Varennes, R. «informatique 1971: principales tendances dans le domaine de l'automatisation des fonctions bibliothéconomiques". Bulletin de l'ACBLF, vol. 17, no 2 (juin 1971), 54-60.

De Varennes, R. "L'ordinateur dans le monde des bibliothèques. Quelques réflexions sur le sujet». Bulletin des bibliothèques de France, vol. 14, nos 9-10 (1969), 377-385

De Varennes, R. et Leclerc-Gauthier, R. Onze ans d'informatique à la Bibliothèque de l'Université Laval. Québec, Bibliothèque, Université Laval, 1975. $50 \mathrm{p}$

Guilmette, P. «La diffusion sélective de l'information appliquée au dépistage et au choix de la documentation", in Stage pratique en informatique documentaire. Rapport. Montréal, 23-25 octobre 1974. Montréal, Corporation des bibliothécaires professionnels du Québec, 1975, 155-165.

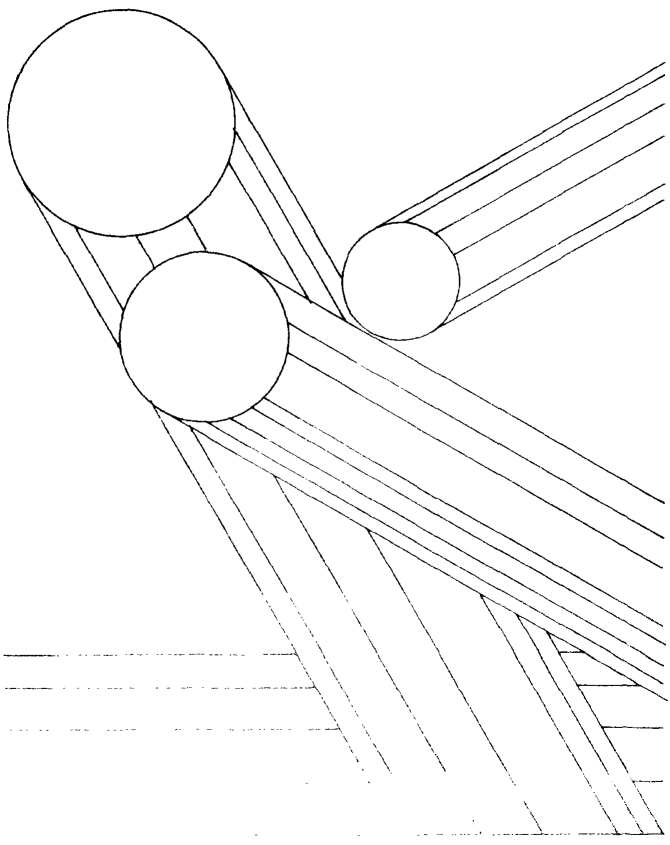

Dans la collection "Histoire

économique et sociale du Canada français»

FRANÇOIS-ÉTIENNE CUGNET

(1719-1751) Entrepreneur et entreprises en Nouvelle-France

par Cameron NISH

XXXII -200 pages. Illustrations.

Relié $\$ 10.00$

Dans la collection "Vies canadiennes»

\section{HONORE MERCIER ET SON TEMPS}

par Robert RUMILLY

Tome I - 1840-1888, 432 p. III.

Tome II - 1888-1894, 432 p. III. $\$ 25.00$

Dans la collection "Satellite 2000"

\section{LES BACTÉRIES, CES BONS MICROBES}

par Denis LAPIERRE 48 pages. Photos de D. Sabourin $\$ 2.00$

Dans la collection:

"L'Église aux quatre vents"

\section{L'ÉVANGÉLISATION DANS LE MONDE MODERNE}

\section{S.S. Paul VI}

104 pages. Exhortation apostolique comparable à une encyclique.

$\$ 1.50$

Hors série:

\section{LE CONTE LITTÉRAIRE QUÉBÉCOIS AU XIXe SIĖCLE}

Essai de bibliographie critique et analytique

par Aurélien BOIVIN

Préface de Maurice Lemire.

385 pages.

$\$ 15.00$

\section{En vente partout} et à la librairie générale

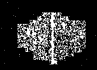

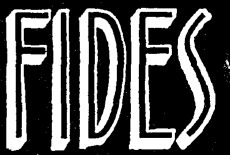

235 est, boul. Dorchester, Montréal H2X 1 N9 tél.: 861-9621 